\title{
CANCER IMMUNOLOGY
}

\section{Eryati Darwin, Faculty of Medicine Andalas University- Padang}

\section{INTRODUCTION}

The immune system can be divided into two parts based on how specific their functions are. These two divisions are called the innate immune system and the adaptive immune system. Innate immune system or natural immunity is the first line defense and nonspecific. It does not require a previous encounter with a microorganism or other invader to work effectively. It responds to invaders immediately, without needing to learn to recognize them. Several types of white blood cells are involved: phagocytes ingest invaders, natural killer cells are formed ready to kill cells that are infected with certain viruses and cancer cells, professional antigen-presenting cells help $\mathrm{T}$ lymphocytes recognize invaders, and some white blood cells release substances involved in inflammation and allergic reactions, such as histamine. These cells often act on their own to destroy invaders. Adaptive immune system is also called acquired specific immunity, it is activated when the innate or non-specific immune system can not efficiently destroy the foreign organism. Specific immunity is distinguished by its specificity for an invading organism. B and $\mathrm{T}$ lymphocytes encounter an invader, learn how to attack it, and remember the specific invader so that they can attack it even more efficiently the next time they encounter it (Janeway,2008).

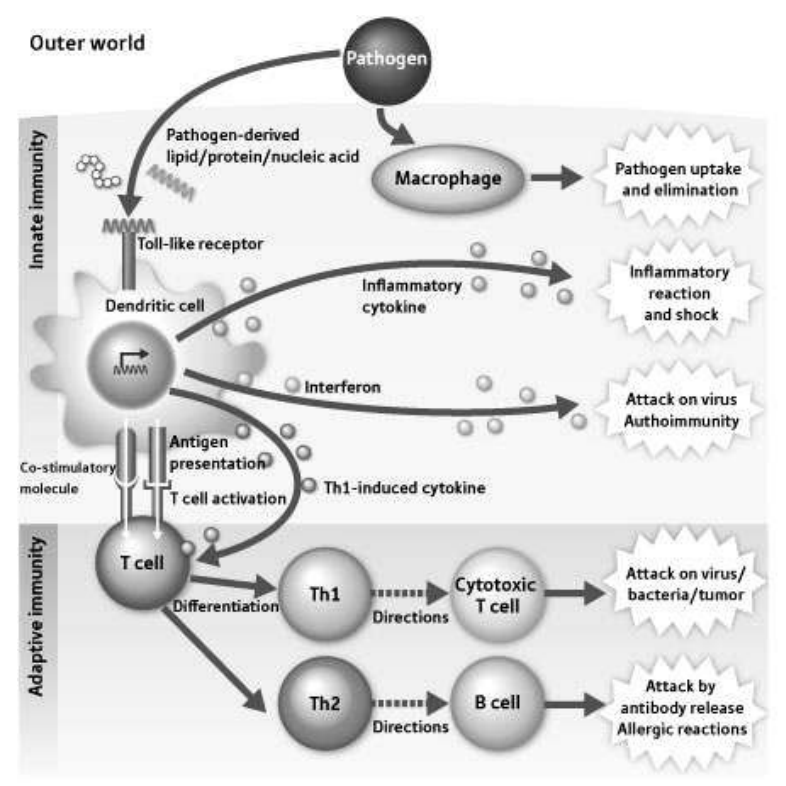

Figure 1: Innate and adaptive immune system. Innate immunity distinguishes between "self" and "non-self", which include phagocytosis, and inflammation. In adaptive immune system, cytotoxic T cells attacs and kills infected or tumour cells, and B cells, which produce antibodies attached to the cells surface, will then bind to an antigen floating around in the blood (Wood, 2006).

Specific immunity takes time to develop after the initial encounter with a new invader because the lymphocytes must adapt to it. Thereafter, response is quick. B and T lymphocytes work together to destroy invaders. Some of these cells do not directly destroy invaders but instead enable other white blood cells to recognize and destroy invaders. 
There are two types of specific immune responses: humoral and cellular. Humoral immunity is mediated by B lymphocytes, which derive from stem cells in the bone marrow. After maturation, these B cells produce antibodies, there are important in combating acute bacterial infections. Cellular immunity is mediated by $\mathrm{T}$ lymphocytes which are processed in the thymus, only those cells, which do not attack self are released. The cellular immune system combats intracellular organisms, performs tumor surveillance, mediates transplant rejection, and fights fungal and viral infections, and also mediate the delayed type hypersensitivity reaction (fig 1). They are distinguished from other types of lymphocyte by the presence of special molecules on their cell surface called T cell receptors (TCR). TCR's are responsible for recognizing and binding to antigens (Wood, 2006).

The immun system consist of protein, cells ad organs that are concerned with defence of the individual, primarily against the threat of desease caused by infectious organisms. Many viruses, bacteria, fungi, yeasts and parasites can cause desease is called a pathogen. However, not all infectious organisms cause desease and some are actually beneficial, for example living bacteria in the gut that help certain foods dogestion, and there are called commensal organisms.

The immune system does not always function properly, sometimes, it reacts to the wrong thing (autoimmunity), other times, it reacts too much (hypersensitivity), and sometimes it simply does not react at all (immunosuppression and immunodeficiency). There are thousands of components to the immune system, and during the course of learning about some of these it can appear that the immune system is far more complicated than necessary for achieving what is on the surface, the simple task of eliminating an infectious organism. There are number of reasons, why the immune system is complex. There are desirability of eliminating pathogens without causing damage to the host that is recognition. The immune system must be able to distinguish between pathogens and host cells so that it can direvt its destructive power towards the pathogens. Many of the specialised features of the immune system are involved in recocnition of foreign pathogens. An additional problem facing the immune system is that pathogens come in all shapes and sizes, with differens lifestyles and different ways of causing diseases (de la Cruz-Merino et al.,2008).

The immune system primarily involved in defence against infectious organisms, but it also plays a role in various othe diseases. The immune system also responsible for rejection of transplant. In this instance the immune system is acting in normally in trying to defend the body against a foreign invader, even though the invader is benevicial. Rejection of transplants is a major cause of graft loss and much effort is being devoted to try to prevent transplant rejection. Evidence is accumulating that the immune system can provide protection aginst some tumors. Exciting new developments also suggest that immune responses can be induced against tumors that normally do not provoke an immune response.

\section{IMMUNE RESPONSE TO TUMORS}

The immune system is an active and effective cancer "gatekeeper." When normal cells turn into cancer cells, some of the antigens on their surface change, then antitumor immune responce activated primarily the innate immune system through circulating effector cells, particularly natural killer cells, cytotoxic $\mathrm{T}$ cells, neutrophils, and macrophages. According to one theory, patrolling cells of the immune system provide continuing bodywide surveillance, spying out and eliminating cells that undergo malignant transformation. Tumors develop when the surveillance system breaks down or is overwhelmed. Antigen-specific B and $\mathrm{T}$ lymphocytes comprise adaptive immunity, which is rather more specific and has the 
ability to generate memory cells. Several mechanisms have been proposed for tumor evasion of the immune recognition process, acting locally and systematically in the tumor microenvironment. Cancer development could be described as the success of the tumor's evasive mechanism over antitumor immune forces. Cancer is an abnormal immune system tolerance to uncontrolled cells, that are usually recognized as tumor cells and are eliminated occasionally escape this control for several reasons such as self-antigens and self-tolerance (fig.2). The knowledge of, and interference with, the mechanisms that allow this "tumor immune tolerance" is the objective of cancer immunotherapy (Volmers and Brändlein, 2007).

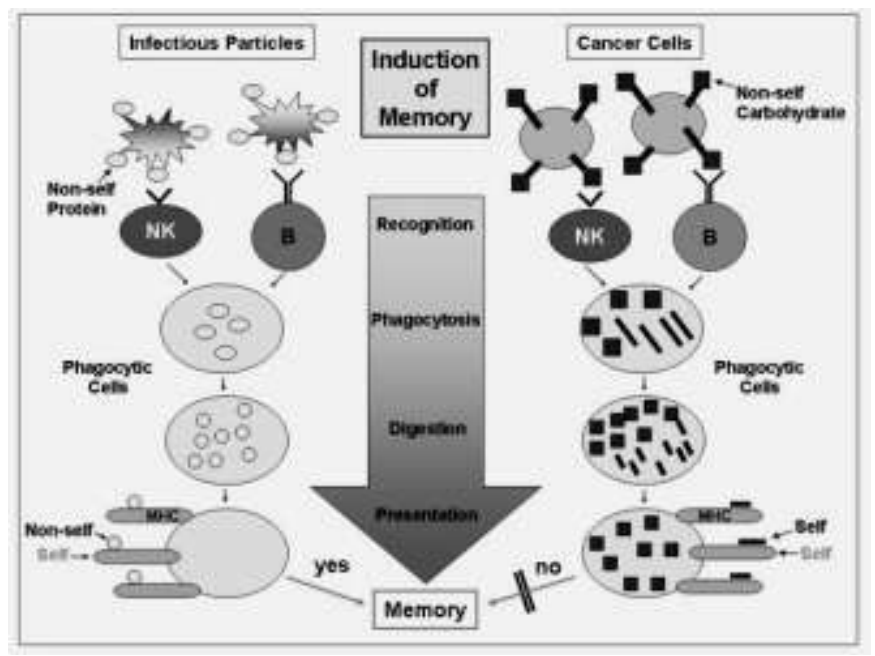

Figure 2: The diferenciation between immune response to infectious particles with protein epitop as nonself with cancer cells with carbo-epitopes. The recognition fails because the phagocytic cells cannot present carbohydrat structures originally seen by the innate immunity. Peptides which are associated with carbohydrate structures are "self" structures and therefore, an education and memory does normally not occur (Volmers and Brändlein, 2007).

\subsection{Innate Immune Response to Tumors}

The function of effector cells of the innate immune system, including natural killer (NK) cells and macrophages.

\section{Natural killer cells}

Natural killer (NK) cells are bone marrow derived lymphocytes originally identified by their large granular morphology and their ability to spontaneously lyse certain tumor targets in vivo and in vitro without prior sensitization. NK cells are also able to eliminate metastatic cells in the circulation. Natural killer cells have been initially identified as a lymphoid population representing the $10-20 \%$ of PBMC, able to lyse MHC class I (MHC-I)negative tumor and virus-infected cells and to orchestrate innate immunity of the organism. The majority of NK cells is localized in peripheral blood, lymph nodes, spleen, and bone marrow but can be induced to migrate toward inflammation sites by different chemoattractants. NK cells constitutively express a lytic machinery able to kill target cells independently from any previous activation. These functional features have suggested since the time of their identification a role for NK cells in the control of tumor growth and metastasis diffusion in vivo (Anderson,2005). 
NK cells mediate their protective effect in two ways: cytotoxicity and cytokine production. Cytotoxicity in NK cells (and cytotoxic T lymphocytes) is carried out by granule exocytosis of membrane pore-forming molecules (perforin) and proteases (granzymes) or death ligand interactions (FasL and TRAIL). Upon target-cell binding, NK cells will also produce large amounts of cytokines, such as interferon- $\gamma$ (IFN- $\gamma)$, TNF- $\alpha$, and granulocytemacrophage colony-stimulating factor. Natural and induced mutations of cytokine (IFN- $\gamma$ and IL-2/IL-15Rb) and cytotoxicity-associated genes (perforin, granzyme A and B, FasL) that are expressed by NK cells also negatively affect antiviral responses and the rejection of tumors (Robertson,2002)

\section{Macrophages}

The role of macrophages in anti-tumor immunity is largely infered from demonstration that activated macrophages kan kill major tumor cells more effeciently than they can kill normal cells. The mecanisms include direct recognition of some surface antigens on tumor cells and activation of macrophages by IFN- $\gamma$, produced by tumor-specific cells. Macrophages can kill tumor cells by several mechanisms, probably the sames as the mechanisms of macrophage killing of infecttious organisms. These mechanisms include the relese of lysosomal enzymes, reactive oxygen species, and nitric oxide. Activated macrophages also produce the cytokine tumor necrosis factor (TNF), which was first characteristized as an agent that can kill tumors mainly by inducing thrombosis in tumor blood vessels.

\subsection{Adaptive Immune Response to Tumors}

Both cellular immunity or $\mathrm{T}$ cell-mediated immunity and humoral immunity occurs spontaneusly to tumors, and some evidence suggest that the $\mathrm{T}$ cell responses play a protective role. Particular efforts are being made to enhance these $\mathrm{T}$ cells responses in immunotherapeutics

\section{Cellular Immunity}

$\mathrm{T}$ lymphocyte is the primary cell thought to be responsible for direct recognition and killing of tumor cells by CD $8^{+}$CTLs. Specific $\mathrm{T}$ lymphocyte carry out an immunologic surveillance, and directly kill autologus tumor cells after recognizing tumors associated antigens (TAAs). T cells are uniquely capable of killing cells expressing TAA intracellularly because peptide fragments derived from these intracellular proteins can be bound to class I $\mathrm{MHC}$ antigens on the tumor cell surface, which can be recognized by cell-surface T-cell receptors. Thus, antigens recognized by cytotoxic T-lymphocytes (CTLs) do not have to be cell-surface proteins, but can be intracellular or even intranuclear proteins. These molecules may represent ideal immunotherapeutic targets because they may be directly involved in the dysregulation of cell growth associated with cancer development. NK cells appear to recognize certain common features of tumor cells, particularly low levels of class I MHC molecules. Some $\mathrm{T}$ cells require the presence of humoral antibodies directed against the tumor cells (antibody-dependent cellular cytotoxicity) to initiate the interactions that lead to the death of tumor cells. The $\mathrm{CD}^{+}$helper $\mathrm{T}$ cells may play a role in anti-tumor immune responses by providing cytokines, such as TNF and IFN- $\gamma$, that can increase tumor cells class I MHC expression and sensitivity to lysis by CTLs (Sulivan et al.,2012).

Other $\mathrm{T}$ cells, termed suppressor $\mathrm{T}$ cells, inhibit the production of an immune response against tumors. The antigen-presenting cell is also key in immune response induction. In addition to diverse cell populations, lymphokines produced by immune cells stimulate growth or induce activities of other immune cells. Such lymphokines include 
interleukin-2 (IL-2), also known as T-cell growth factor, and IFN. Recently described growth factors, such as interleukin-12 (IL-12), specifically induce CTLs rather than suppress T-cell responses and thus enhance antitumor immune responses.

\section{Humoral Immunity}

Tumor-bearing host may produce antibodies against various tumor antigens. Antibodies may kill tumor cells by activating complement or by antibody-dependent cell mediated cytotoxicity, in which Fc receptor-bearing macrophages or NK cells mediate the killing. Cytotoxic antibodies are complement-fixing in general and are directed against surface antigens of relatively high density. IgM antibodies usually are more cytotoxic in transplantation systems than are IgG antibodies. The ability of antibodies to eliminate tumor cells has been demonstrated invitro such as antibodies specific for oncogenic virus HPV, can prevent virally induced tumors.

Humoral antibodies directed against human tumor cells or their constituents have been shown in vitro in the sera of patients with Burkitt's lymphoma, malignant melanoma, osteosarcoma, neuroblastoma, and lung, breast, and GI carcinomas. However, humoral antibody-mediated protection against tumor growth in vivo is demonstrable only in certain animal leukemias and lymphomas. In contrast, lymphoid cell-mediated protection in vivo occurs with many animal tumors (Reuschenbach et al., 2009).

\section{IMMUNO SURVEILLANCE}

The immune system has three primary roles in the prevention of tumors. First, the immune system can protect the host from virus-induced tumors by eliminating or suppressing viral infections. Second, the timely elimination of pathogens and prompt resolution of inflammation can prevent the establishment of an inflammatory environment conducive to tumorigenesis. Third, the immune system can specifically identify and eliminate tumor cells on the basis of their expression of tumor-specific antigens or molecules induced by cellular stress (Igney and Krammer, 2002).

The ability of immune system to identify and eliminate tumor cells is referred as tumor immune surveillance, whereby the immune system can recognize and eliminates transformed or pre-cancerous and/or cancerous cells before they can cause harm. Cancer immunosurveillance appears to be an important host protection process that inhibits carcinogenesis and maintains regular cellular homeostasis. It has also been suggested that immunosurveillance primarily functions as a component of a more general process of cancer immunoediting (Swann et al.,2007). The process of cancer immunoediting is occured in three phases and called as three Es of cancer immunoediting: 1. Elimination corresponds to immunosurveillance, 2. Equilibrium, represents the process by which the immune system iteratively selects and/or promotes the generation of tumor cell variants with increasing capacities to survive immune attack. 3. Escape is the process wherein the immunologically sculpted tumor expands in an uncontrolled manner in the immunocompetent host (fig.3).

The first phase, of cancer immunoediting is the elimination phase (formerly known as cancer immunosurveillance), consists of the recognition of tumor cells by the innate and the adaptive immune system, leading to the killing of these cells. This is followed by the induction of inflammatory signals which is essential for recruiting cells of the innate immune 
system such as NK cells, cytotoxic T cells, macrophages and dendritic cells to the tumor site. During this phase, the cells are stimulated to produce IFN- $\gamma$ that induces tumor death as well as promoting the production of chemokines CXCL10, CXCL9 and CXCL11. These chemokines play an important role in promoting tumor death by blocking the formation of new blood vessels. The recruitment of more immune cells also occurs and is mediated by the chemokines produced during the inflammatory process.

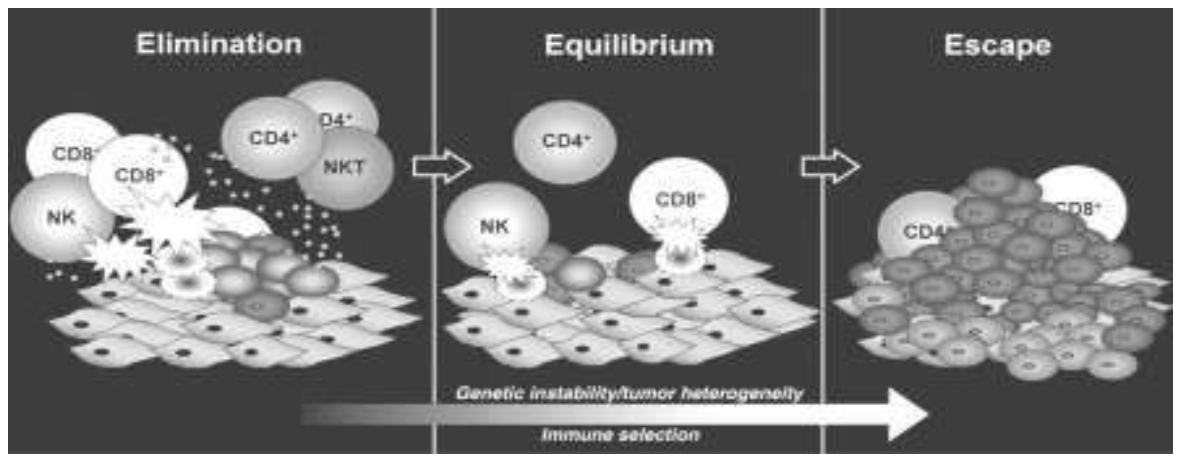

Figure 3: Immunoediting, ability of the immune system to detect tumor cells, destroy them and finaly escape from it (Dunn et al., 2004)

Natural killer cells and macrophages transactivate one another via the reciprocal production of IFN- $\gamma$ and IL-12. This again promotes more tumor killing by these cells via apoptosis and the production of reactive oxygen and nitrogen intermediates. In the draining lymph nodes, tumor-specific dendritic cells trigger the differentiation of Th1 cells which in turn facilitates the development of $\mathrm{CD} 8+\mathrm{T}$ cells. In addition, $\mathrm{CD} 4^{+}$and $\mathrm{CD}^{+} \propto \beta \mathrm{T}$ cells recognize tumor-specific or tumor-associated antigens (TAA) in the context of MHC class II and class I molecules, respectively, and B cells produce antibodies that recognize antigens at tumor-cell surfaces. Recognition by innate and adaptive immune mechanisms leads to direct killing of tumor cells, as well as production of chemokines and other cytokines that facilitate killing of tumor cells. If some tumor cells are not killed in the elimination phase, then the process can progress to the second phase

The equilibrium phase, a subclinical phase in which the tumour persists but is prevented from expanding by immune pressure. In this phase, lymphocytes and IFN-gamma exert a selection pressure on tumor cells which are genetically unstable and rapidly mutating. Tumor cell variants which have acquired resistance to elimination then enter the escape phase. In this phase, tumor cells continue to grow and expand in an uncontrolled manner and may eventually lead to malignancies.

The third or final phase is the escape fase, its begins when the balance between the immune response and the tumor tilts towards tumor growth as a result of immune exhaustion or inhibition or as a result of the emergence of tumour-cell variants that enable the tumour to evade immune pressure. Non-immunogenic transformed cells directly enter the escape phase. This final phase occurs when some tumor cells have accumulated sufficient mutation with the appearance of clinically detectable, progressively growing tumours (Dunn et al., 2002; Dunn et al., 2006; DuPage et al.,2012) 


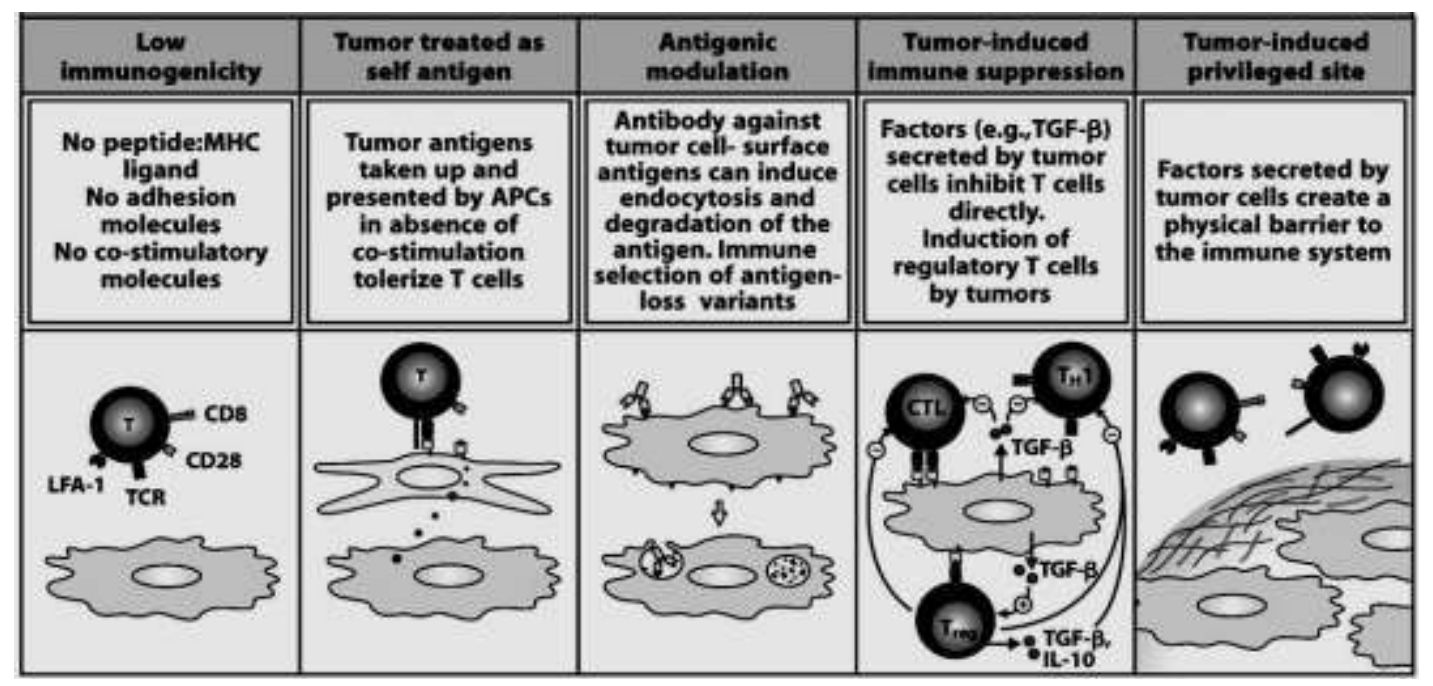

Figure 4: Mechanisms by which tumors avoid immune recognition (Janeway,2008)

According to the Immune Surveillance Theory, cancer cells that arise in the body are eliminated by the immune system. However, due to impaired immune reactivity, cancer cells may escape destruction. Tumors evade immune recognition by several mechanisms. First panel: the reason for failure of immune surveillance may be the fact that in the early development of a tumor, the amount of antigen may be too small to stimulate the immune system (low immunogenicity). Some tumors do not have peptides of novel proteins that can be presented by MHC molecoles, and therefore appear normal to the immune system. Second panel: Tumor antigens presented in the absence of co-stimulatory signals will make the responding $\mathrm{T}$ cells tolerant to the antigen. Tumors may not express neo-antigens that are immunogenic or they may fail to express co-stimulatory molecules required for the activation of $\mathrm{T}$ cells. In addition, certain tumors are known to lack or be poor expressers of MHC antigen. or, due to the rapid proliferation of malignant cells (high dose tolerance), the immune system is quickly overwhelmed (panel three). In addition, some tumors may evade the immune system by secreting immunosuppressive molecules suchb as TGF $\beta$ and others may induce regulatory cells particularly the CD4+CD25+ FoxP3+ T regulatory cells. Also, some tumors may shed their antigens which in turn may interact and block antibodies and $\mathrm{T}$ cells from reacting with the tumor cells. Tumor cells secrete collagen that form a physical barrier around the tumor, preventing lymphocyte acces (fig 4) (Janeway,2008; Disis,2010).

\section{TUMOR ANTIGENS AND TUMOR IMMUNODIAGNOSIS}

The majority of tumor antigens are also present on normal cells and are referred to as tumor associated transplantation antigens. They may be expressed at higher levels on tumor cells when compared to normal cells. Alternatively, they may be expressed only during development of cells and lost during adult life but re-expressed in tumors. A variety of tumor antigens that may be recognized by $\mathrm{T}$ and $\mathrm{B}$ lymphocytes have been identified in human and animal cancers. It is important to identify tumor antigens in human because they may be used as component of tumor vaccines, and antibodies and effector $\mathrm{T}$ cells generated against these antigens may be used for immunotherapy.

In order for the immune system to react against a tumor, the latter must have antigens that are recognized as foreign. A number of alterations in gene expression occur in cells during tumorigenesis. Tumorigenesis may lead to expression of new antigens (neoantigens) 
or alteration in existing antigens that are found on normal cells. These antigens may include membrane receptors, regulators of cell cycle and apoptosis, or molecules involved in signal transduction pathways. There are 2 main types of tumor antigens:

1. Tumor-specific transplantation antigens (TSTA) which are unique to tumor cells and not expressed on normal cells. They are responsible for rejection of the tumor.

2. Tumor associated transplantation antigens (TATA) that are expressed by tumor cells and normal cells.

Although chemical, UV or virus-induced tumors express neo-antigens, the majority of these tumors are often weakly immunogenic or non-immunogenic. In most cases, TSTAs cannot be identified easily. Some of these antigens may be secreted while others may be membrane-associated molecules. Tumor-associated developmental antigens or onco-fetal antigens, include $\alpha$-fetoprotein (AFP) and carcino-embryonic antigen (CEA) found secreted in the serum. AFP a normal product of fetal liver cells, is also found in found in the sera of patients with hepatocellular carcinoma, yolk sac neoplasms, and, frequently, ovarian or testicular embryonal carcinoma. CEA is a protein-polysaccharide complex on cell membranes and in secreted fluids. Its found in colon cancer and in normal fetal intestine, pancreas, and liver and these are important in diagnosis (Peakman and Vergagni, 2004).

TAAs can be useful tumor markers in the diagnosis and management of various tumors. An ideal tumor marker is released only from tumor tissue, is specific for a given tumor type (to direct diagnostic assessment), is detectable at low levels of tumor cell burden, has a direct relationship to the tumor cell burden and the marker concentration in blood or other body fluid, and is present in all patients with the tumor. Most tumors release antigenic macromolecules into the circulation that can be detected by immunoassay. Although useful in monitoring patients for tumor recurrence after therapy, no tumor marker has undisputed specificity or sensitivity for application in early diagnosis or mass cancer screening programs.

$\beta$ Subunit of human chorionic gonadotropin ( $\beta-\mathrm{HCG})$, measured by immunoassay, is the major clinical marker in women with gestational trophoblastic neoplasia (GTN)-a disease spectrum that includes hydatidiform mole, nonmetastatic GTN, and metastatic GTN - and in about $2 / 3$ of men with testicular embryonal or choriocarcinoma. The $\beta$ subunit is measured because it is specific for HCG.

Prostate-specific antigen (PSA), a glycoprotein located in ductal epithelial cells of the prostate gland, can be detected in low concentrations in the sera of healthy men. Using an appropriate upper limit of normal, assays with monoclonal antibodies detect elevated serum levels of PSA in about $90 \%$ of patients with advanced prostate cancer, even in the absence of defined metastatic disease. It is more sensitive than prostatic acid phosphatase. However, because PSA is elevated in benign prostatic hypertrophy, it is less specific. PSA can be used to monitor recurrence after prostatic carcinoma has been diagnosed and treated (Abas et al.,2007).

\section{ANGIOGENESIS AND TUMOR GROWTH}

Angiogenesis is the process through which new blood vessels form and grow. Angiogenesis is a natural physiological function which can be subverted by cancer cells to satisfy their increasing need for nutrients and oxygen as tumors grow untamed. In tumor growth lymphatic vessels also growth, called lymphangiogenesis. Both have an essential role in the formation of a new vascular network to supply nutrients, oxygen and immune cells, and also to remove waste products. Solid tumor growth and survival, ultimately critically 
depend on parallel proliveration of endothelial cells constituting the tumor blood vesesels network (fig.5). Angiogenic and lymphangiogenic factors are increasingly receiving attention, especially in the field of neoplastic vascularization (Siemann,2002)

Cancer has the ability to spread to adjacent or distant organs, which makes it life threatening. In the course of their migration, inflammatory cells secrete additional substances that intensify the angiogenic call. Tumor cells can penetrate blood or lymphatic vessels, circulate through the intravascular stream, and then proliferate at another site, called metastasis. For the metastatic spread of cancer tissue, growth of the vascular network is important (Prior et al.,2004).
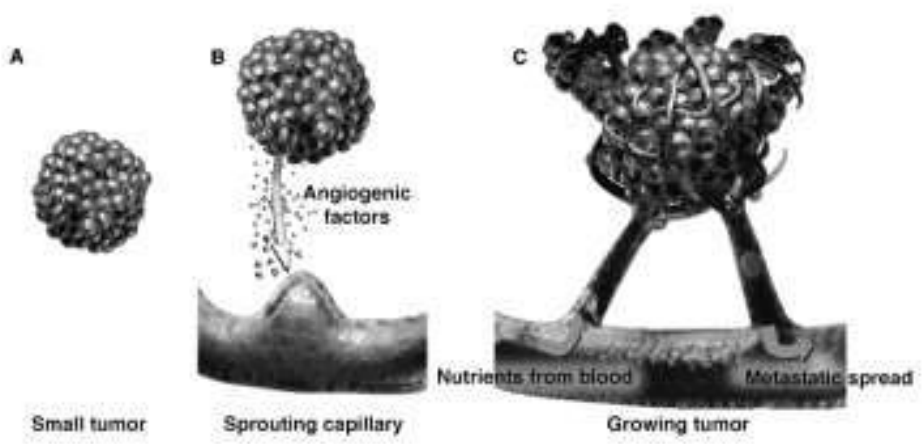

Figure 5: Tumor angiogenesis and neovasculature (Siemann, 2002)

A variety of different growth factors and cytokines can promote angiogenesis, but one of the most important is vascular endothelial growth factor (VEGF). VEGF is a soluble protein secreted by most types of cells, excepted by endothelial cells themselves. On the other hand, endothelial cells express receptors for this growth factor. Hypoxic conditions, as seen in cancer growth, are a potent trigger of VEGF expression. Interaction of VEGF with its receptor induces an intracellular signaling cascade that favors endothelial cell survival, proliferation and migration and increases vascular permeability. VEGF also up-regulates the expression of other proteins involved in angiogenesis. High serum levels of VEGF correlates with poor prognosis in cancer patients (Nishida et al.,2006).

\section{EFFECT OF AGING AND STRESS TO CANCER}

The effect of stressful experience (include physical stressor such as pathogens and toxins, and phsychological stressor such as majir life events, trauma, abuse, or factors related to the environtment in the home, workplace, family, or neighbourhood), on various parts of immunological function and the association with cancer have been investigated in transverse and longitudianal prospective studies. Little is known about why susceptibility to cancer is so variable. Cancer risk increases with age, one factor may be that aging lymphocytes accumulate genetic errors that decrease effectiveness, as well as the thymus function declining with age and with a decrease in cell-mediated immune competence. It has also been proven that tumors grow faster in stressed animals.

The end result of our chronically stressed-out brains sending stimulatory signals to adrenal glands to produce stress hormones (like cortisol and epinephrine.) Long-term exposure The stress hormones causes: increase the production of free radicals that cause DNA damage and impaired immune function; increase inflammation through the production 
of inflammatory proteins (cytokines) that impair immune function and promote cancer growth; reduce the ability of abnormal cells to undergo apoptosis (cell death) and DNA repair, important self-regulating anticancer mechanisms, and stimulate the production of insulin-like growth factor-1 (IGF-1)), vascular endothelial growth factor(VEGF) and other growth factors that can promote tumor cell growth (Reiche et al.,2004).

\section{CANCER AND INFLAMMATION}

An association between the development of cancer and inflammation has long-been appreciated. Inflammation can cause cancer and otherwise, cancer can cause inflammation. The inflammatory response orchestrates host defenses to microbial infection and mediates tissue repair and regeneration, which may occur due to infectious or non-infectious tissue damage. Chronic infection in immunocompetent hosts such as human papilloma virus or hepatitis B and C virus infection leads to cervical and hepatocellular carcinoma, respectively. Cells have intrinsic mechanisms by which to prevent unregulated proliferation or the accumulation of DNA mutations. These include tumor suppressor pathways that mediate DNA repair, cell cycle arrest, apoptosis and senescence. In the face of DNA damage or oncogenic activation, cells will either repair their DNA and prevent mutations or initiated cells will undergo cell death.

Pre-malignant tumors are "wound-like", whereas in the first phase, the body treats early tumors as wounds. This phase is characterized by tumor growth mediated by the actions of the stroma "indirect control" as occurs in physiologic tissue repair. Bone-marrow derived cells, including mast cells, are responsible for providing matrix metalloproteases which convert VEGF into a biological active form to stimulate the pro-tumorigenic angiogenic switch. However, during later tumor growth, it appears pro-inflammatory factors, such as MMPs, come under direct control by the tumors themselves. COX-2 is expressed by stromal cells in early tumor, perhaps as part of a response to early tumor associated wounding; in larger tumors, COX-2 is expressed by the dysplastic epithelium itself (RakoffNahoum,2006).

\section{IMMUNOTHERAPY}

Cancers are caused by the progressive growth of the progeny of a single transformed cell. Therefore, curing cancer requires that all the malignant cells be removed or destroyed without killing the patient. In the nineteenth century it was noticed that some individuals with cancer showed a temporary improvement during bacterial infection, but treatment with bacteria cause more chance killing the patients than helping them. In twentieth century the genetic cancer ad immune surveillance was born. An attractive way to achieve this would be to induce an immune response against the tumor that would discriminate between the cells of the tumor and their normal cell counterparts. Immunological approaches to the treatment of cancer have been attempted for over a century, with tantalizing but unsustainable results. Experiments in animals have, however, provided evidence for immune responses to tumors and have shown that $\mathrm{T}$ cells are a critical mediator of tumor immunity. More recently, advances in our understanding of antigen presentation and the molecules involved in T-cell activation have provided new immunotherapeutic strategies based on a better molecular understanding of the immune response. These are showing some success in animal models and are now being tested in human patients (de la Cruz-Merino, 2008). 


\subsection{Stimulation of active host immune responce to tumor}

Cancer can be prevented with active immunization, and there are many different types of cancer vaccine formulations in use today, each designed to optimize the ability to immunize against self-proteins. The earliest attemps to boost anti-tumor immunity relied on nonspecific immune stimulation.

\section{Vaccination with tumor cells and tumor antigens}

More recently, vaccins composed of killed tumor cells or tumor antigens have been administered to patients, and strategies for enhancing immune responses against the tumor are being developed. Studies have shown that vaccination against human papillomavirus (HPV) type 16 is highly effective in preventing the development of cervical cancer. The other vaccines such as adenovirus, melanoma, plasmids dendritic cells and cytokine vaccines.

Augmentation of host immunity to tumors with costimulators and cytokines

Two potential approaches for boosting host responses to tumors are to artiviacially provide costimulation for tumor-specific $\mathrm{T}$ cells and to provide cytokines that can enhance the activation of tumor-specific T cells, particularly $\mathrm{CD}^{+} \mathrm{CTLs}$. Many cytokines also have the potential to induce nonspecific inflammatory response, which by themselves may have anti-tumor activity. Tumor cells may be transfected with cytokine gene such as IL-2, IL-4, IFN- $\gamma$, ar granulocyte-macrophage colony-stimulating factor (GM-CSF) to localize the cytokine effect to where they are needed. The local production of cytokinec may augment $\mathrm{T}$ cells reponse to tumor antigens, and cytokine-expressing tumors may act as effective tumor vaccines. Cytokines may also be administered systematically for the trearment of various human tumors.

\section{Blocking inhibitory pathways to promote tumor immunity}

Immunotherapy strategy is based on the inhibitory receptor for B7, called CTLA-4 that maintaining self-tolerance. The depletion of regulatory $\mathrm{T}$ cells may also enhance antitumor immunity.

\section{Nonspecific stimulation of the immune system}

Nonspecific immune stimulation of patients with tumors by injection of inflammatory substances such as bacillus Calmette-Guérin (BCG) at the sites of tumor growth has been tried for many years. The BCG mycobacteria activate macrophages and thereby promote macrophage-mediated killing of the tumor cells. In addition, teh bacrteria function as adjuvants and may stimulate $\mathrm{T}$ cell responses to tumor ntigens. Another approach is to administer activating anti-CD3 antibodies to prevent of tumor growth.

\subsection{Passive immunotherapy for tumor}

Passive immunotherapy involves the transfer of immune effectors, including antibodies, tumor-specific $\mathrm{T}$ cells or other immune cells and other factors into patients or host. Pasive immunization against tumors is rapid but does not lead to long-lived immunity. Several approaches to passive immunotherapy are being tried, with variable success.

\section{Antibodies against tumor cells}

Anti-tumor antibodies are specific monoclonal antibodies, that produced by fusion of animals tumor imunized B cells with myeloma cells, called a hybridoma. There are two variation of monoclonal antibodies as therapeutic agents of cancer. One is to use unmodified antibodies to eradicate tumors by the same effector mechanisms that are use to eliminate microbes, including opsonization, phagocutosis, activation of complement system or the 
involvement of antibody-dependent cytotoxicity (e.g. Her2/Neu for treatment of breast cancer, antibodies agaist CD20).

A second approach on ani-tumor antibodies have been tried to improve their effectiveness. Tumor-specific antibodies may be coupled to toxic molecules, radioisotopes, and anti-tumor drugs to promote the delivery of these cytotoxic agents to the tumor ( e.g. anti-CD20 conjugated to Pseudomonas toxin or ricin toxin).

\section{Adoptive cellular therapy}

Adoptive cellular imunotherapy is the transfer of cultured immune cells that have anti-tumor reactivity into a tumor-bearing host. The cells can be transferred are expanded from the lymphocytes of patients with the tumor. Adoptive Transfer of lymphocytes such as lymphokine-activated killer (LAK) cells which are IL-2 activated T and NK cells, Tumorinfiltrating lymphocytes (TIL) or NK cells and dendritic cells (Zamai et al.,2007).

\subsection{Antiangiogenesis as a strategy against cancer}

Antiangiogenic therapy is likely to deliver lower doses of targeted, less toxic drugs over longer periods of time, whereas chemotherapy delivers high doses of toxic drugs in a short period of time. Chemotherapy destroys both normal and cancerous cells in days or weeks, while targeted antiangiogenic therapies may take long periods to achieve their full benefit. Researchers are now exploring whether inhibiting angiogenesis can slow down or prevent the growth and spread of cancer cells in humans. The malignant tissue would be deprived of its oxygen and nutrient supply, as well as be unable to eliminate metabolic wastes. This in turn would inhibit tumor progression and metastatic progression that accompanies most advanced cancers (Shim et al.,2007). These are the main steps of the angiogenic process that can be interrupted:

1. Inhibiting endogenous angiogenic factors, such as bFGF (basic Fibroblast Growth Factor) and VEGF

2. Inhibiting degradative enzymes (Matrix Metalloproteinases) responsible for the degradation of the basement membrane of blood vessels

3. Inhibiting endothelial cell proliferation and endothelial cell migration

4. Inhibiting the activation and differentiation of endothelial cells

However, the challenge is to develop an antiangiogenic factor that does not affect the existing vasculature. 


\section{REFERENCES}

1. Abbas AK, Lichtman AH, and Pillai S. Cellular and molecular immunology $6^{\text {th }}$ ed. Saunders Elsevier,Philadelphia,2007

2. Anderson SK. Biology of Natural Killer Cells: What Is the Relationship between Natural Killer Cells and Cancer? Will an Increased Number and/or Function of Natural Killer Cells Result in Lower Cancer Incidence?. J Nutr, 2005; 135.12: 2910-2915

3. de la Cruz-Merino L, Grande-Pulido E, Albero-Tamarit A and de Villena MEC. Cancer and Immune Response: Old and New Evidence for Future Challenges. The Oncologist, 2008; 13.12: 1246-1254

4. Disis ML. Immune Regulation of Cancer. Journal of clinical oncology, 2010; 28.29: 4531-4538

5. Dunn GP, Bruce AT, Ikeda H, Old LJ, and Schreiber RD. Cancer immunoediting: from immunosurveillance to tumor escape. Nature Immunology, 2002;3.11: 991-998

6. Dunn GP , Old LJ and Schreiber RD. The three Es of cancer immunoediting. Annu Rev immunol, 2004; 22;329-360

7. Dunn GP, Koebel CM and Schreiber RD. Interferons, immunity and cancer immunoediting. Nature Reviews Immunology, 2006; 6: 836-848

8. DuPage M, Mazumandar C, Schmidt LM, Cheung AF and Jacks T. Expression of tumour-specific antigens underlies cancer immunoediting. Nature, 2012; 8; 482.7385: 405-409.

9. Igney FH and Krammer PH. Immune escape of tumors: apoptosis resistance and tumor counterattack. Journal of Leukocyte Biology, 2002; 71.6: 907-920

10. Janeway C. Janeway's Immunobiology $7^{\text {ed }}$. Murphy K, travers.P and Walport M. Garland Science, 2008

11. Kaufman HL and Disis L. Immune system versus tumor: shifting the balance in favor of DCs and effective immunity. J Clin Invest, 2004;113.5:664-667

12. Nishida N, Yano H, Nishida T, Kamura T and Kojiro M. Angiogenesis in Cancer. Vasc Health Risk Manag, 2006; 2.3: 213-219

13. Peakman $M$ and Vergagni D. Basic and clinical immunology. Churchil Livingston. Edinburg, London, Toronto. 2004

14. Prior BM, Yang HT and Terjung RL. What makes vessels grow with exercise training?. Journal of Applied Physiology, 2004; 97. 3: 1119-1128

15. Rakoff-Nahoum S. Cancer and inflammation?. Yale J Biol Med, 2006; 79.3-4: 123130.

16. Reiche EMV, Nunes SOV, and Morimoto,HK. Stress, depression, the immune system, and cancer. The Lancet of Oncology,2004; 5: 617-625

17. Reuschenbach M, von Knebel Doeberitz $M$ and Wentzensen N. A systematic review of humoral immune responses against tumor antigens. Cancer Immunol Immunother. 2009 ; 58.10: 1535-1544.

18. Robertson MJ. Role of chemokines in the biology of natural killer cells. Journal of Leukocyte Biology, 2002; 71.2: 173-183

19. Schreiber RD, Old LJ and Smyth MJ. Cancer Immunoediting: Integrating Immunity's Roles in Cancer Suppression and Promotion. Science, 2011; 331. 6024:1565-1570

20. Shim WSN, Ho IAW and Wo PEH. Angiopoietin: A TIE(d) Balance in Tumor Angiogenesis. Mol Cancer Res, 2007;5: 655

21. Sieman DW. Vascular targeting agent. Horizon in cancer theurapeutics: From bench to bedsise, 2002; 3.2: rom bench to bedsise, 2002; 3.2: -16 
22. Sulivan TO, Konefka RS, Vermi W, Koebel CM, Arthur C, White JM et al., Cancer immunoediting by the innate immune system in the absence of adaptive immunity. Journal of experimental Medicine,2012; 209. 10: 1869-1882

23. Swann JB and Smyth MJ. Immune surveillance of tumors. J Clin Invest. 2007; 117.): 1137-1146.

24. Volmers HP and Brändlein S. Tumors: Too sweet to remember?. Molecular Cancer 2007; 6.78: 4598-6-78

25. Zamai L, Ponti C, Mirandola P, Gobbi G, Papa Sgaleotti L, Cocco L and Vitale M. NK Cells and cancer. The Journal of Immunology, 2007 ;178. 7: 4011-4016 